OPEN ACCESS

Edited by:

Laszlo Otvos,

Olpe LLC, United States

Reviewed by:

Peng Zhang

Shanghai Institutes for Biological

Sciences (CAS), China

Demetres Leonidas,

University of Thessaly, Greece

*Correspondence:

Rosmarie Friemann

rosmarie.friemann@gu.se

Renwick C. J. Dobson

renwick.dobson@canterbury.ac.nz

†These authors have contributed equally to this work.

Specialty section:

This article was submitted to Chemical Biology,

a section of the journal

Frontiers in Chemistry

Received: 28 March 2018

Accepted: 01 June 2018

Published: 04 July 2018

Citation:

North RA, Wahlgren WY, Remus DM,

Scalise M, Kessans SA, Dunevall E,

Claesson E, Soares da Costa TP,

Perugini MA, Ramaswamy $S$,

Allison JR, Indiveri C, Friemann $R$ and Dobson RCJ (2018) The Sodium Sialic

Acid Symporter From Staphylococcus

aureus Has Altered Substrate

Specificity. Front. Chem. 6:233.

doi: 10.3389/fchem.2018.00233

\title{
The Sodium Sialic Acid Symporter From Staphylococcus aureus Has Altered Substrate Specificity
}

\author{
Rachel A. North ${ }^{1,2,3 \dagger}$, Weixiao Y. Wahlgren ${ }^{3,4 t}$, Daniela M. Remus ${ }^{1,2}$, \\ Mariafrancesca Scalise ${ }^{5}$, Sarah A. Kessans ${ }^{1,2}$, Elin Dunevall ${ }^{3}$, Elin Claesson ${ }^{3}$, \\ Tatiana P. Soares da Costa ${ }^{6}$, Matthew A. Perugini ${ }^{6}$, S. Ramaswamy ${ }^{7}$, Jane R. Allison ${ }^{2,8,9}$, \\ Cesare Indiveri ${ }^{5}$, Rosmarie Friemann ${ }^{3,4 *}$ and Renwick C. J. Dobson ${ }^{1,10 *}$ \\ ${ }^{1}$ School of Biological Sciences, University of Canterbury, Christchurch, New Zealand, ${ }^{2}$ Biomolecular Interaction Centre, \\ University of Canterbury, Christchurch, New Zealand, ${ }^{3}$ Department of Chemistry and Molecular Biology, University of \\ Gothenburg, Gothenburg, Sweden, ${ }^{4}$ Centre for Antibiotic Resistance Research, University of Gothenburg, Gothenburg, \\ Sweden, ${ }^{5}$ Unit of Biochemistry and Molecular Biotechnology, Department DiBEST (Biologia, Ecologia, Scienze della Terra), \\ University of Calabria, Arcavacata di Rende, Italy, ${ }^{6}$ Department of Biochemistry and Genetics, La Trobe Institute for Molecular \\ Science, La Trobe University, Melbourne, VIC, Australia, ${ }^{7}$ The Institute for Stem Cell Biology and Regenerative Medicine, \\ Bangalore, India, ${ }^{8}$ Centre for Theoretical Chemistry and Physics, Institute of Natural and Mathematical Sciences, Massey \\ University, Auckland, New Zealand, ${ }^{9}$ Maurice Wilkins Centre for Molecular Biodiscovery, University of Auckland, Auckland, \\ New Zealand, ${ }^{10}$ Department of Biochemistry and Molecular Biology, Bio21 Molecular Science and Biotechnology Institute, \\ University of Melbourne, Parkville, VIC, Australia
}

Mammalian cell surfaces are decorated with complex glycoconjugates that terminate with negatively charged sialic acids. Commensal and pathogenic bacteria can use hostderived sialic acids for a competitive advantage, but require a functional sialic acid transporter to import the sugar into the cell. This work investigates the sodium sialic acid symporter (SiaT) from Staphylococcus aureus (SaSiaT). We demonstrate that SaSiaT rescues an Escherichia coli strain lacking its endogenous sialic acid transporter when grown on the sialic acids $\mathrm{N}$-acetylneuraminic acid (Neu5Ac) or N-glycolylneuraminic acid (Neu5Gc). We then develop an expression, purification and detergent solubilization system for SaSiaT and demonstrate that the protein is largely monodisperse in solution with a stable monomeric oligomeric state. Binding studies reveal that SaSiaT has a higher affinity for Neu5Gc over Neu5Ac, which was unexpected and is not seen in another SiaT homolog. We develop a homology model and use comparative sequence analyses to identify substitutions in the substrate-binding site of SaSiaT that may explain the altered specificity. SaSiaT is shown to be electrogenic, and transport is dependent upon more than one $\mathrm{Na}^{+}$ion for every sialic acid molecule. A functional sialic acid transporter is essential for the uptake and utilization of sialic acid in a range of pathogenic bacteria, and developing new inhibitors that target these transporters is a valid mechanism for inhibiting bacterial growth. By demonstrating a route to functional recombinant SaSiaT, and developing the in vivo and in vitro assay systems, our work underpins the design of inhibitors to this transporter.

Keywords: antibiotic resistance, sialic acids, SiaT, sodium solute symporter, Staphylococcus aureus 


\section{INTRODUCTION}

Mammalian cell surfaces are decorated with complex glycoconjugates, such as glycoproteins and glycolipids. Found at the terminal non-reducing positions of these cell-surface glycoconjugates are negatively charged sialic acids, which mediate a diverse array of cellular interactions, recognition and adhesion.

Sialic acids comprise a large family of nine-carbon acidic monosaccharides, the most common of which is $\mathrm{N}$ acetylneuraminic acid (Neu5Ac). While Neu5Ac is ubiquitously synthesized, the closely related sialic acid, $N$-glycolylneuraminic acid (Neu5Gc) is not. Although Neu5Ac and Neu5Gc sialic acids are widely expressed on mammalian tissues, human cells do not synthesize Neu5Gc. This is because humans have an inactivating mutation in the gene encoding CMP- $N$-acetylneuraminic acid hydroxylase, the rate-limiting enzyme for the generation of Neu5Gc in the cells of other mammals (Varki, 2001).

Mammalian commensal and pathogenic bacteria that colonize sialic acid rich tissues, such as the respiratory or gastrointestinal tract, have evolved mechanisms to use host-derived sialic acids for a competitive advantage; this suggests a link between sialic acid uptake/utilization and survival in vivo (AlmagroMoreno and Boyd, 2009). Some bacteria, such as Haemophilus influenzae (Vimr et al., 2000; Bouchet et al., 2003), and Neisseria meningitides (Vimr et al., 2004) incorporate sialic acid into their cell surface macromolecules to trick the host's innate immune response. Others, such as Escherichia coli (Vimr and Troy, 1985; Chang et al., 2004), Staphylococcus aureus (Olson et al., 2013), and Vibrio vulnificus (Jeong et al., 2009) use a suite of enzymes (North et al., 2013, 2014a,b, 2016) to degrade sialic acids as a source of carbon, nitrogen and energy. Notably, H. influenzae also metabolizes sialic acids in this way, and must make a metabolic decision between cell surface sialylation and sialic acid degradation (Vimr et al., 2000).

Bacteria that import sialic acids have evolved multiple mechanisms of transport across the cytoplasmic membrane. To date, four unique transporter families have been recognized, including those from the ATP binding cassette (ABC) (Post et al., 2005), tripartite ATP-independent periplasmic (TRAP) (Allen et al., 2005), major facilitator superfamily (MFS) (Vimr and Troy, 1985), and sodium solute symporter (SSS) (Severi et al., 2010; Wahlgren et al., 2018) transporter families (North et al., 2017). Whilst most bacteria possess only one type of sialic acid transporter, there are a few exceptions that are predicted to express two family types (Severi et al., 2010). It is not understood why these organisms produce more than one type of sialic acid transporter, but it is possible that they import sialic acid derivatives that are known in biological contexts.

Developing novel inhibitors that target bacterial sialic acid transporters may be a valid mechanism for inhibiting bacterial growth-several lines of evidence support this. It has been shown that a dedicated and functional sialic acid membrane transporter is required for the uptake of sialic acids (Vimr and Troy, 1985; Severi et al., 2005, 2010). Moreover, in vivo mouse studies demonstrate that sialic acid uptake and utilization is essential for colonization and persistence in a range of pathogenic bacteria (Chang et al., 2004; Almagro-Moreno and Boyd, 2009; Jeong et al., 2009; Pezzicoli et al., 2012). Knocking out the respective sialic acid transporter genes in Salmonella enterica serovar Typhirium and Clostridium difficile impairs outgrowth during post-antibiotic expansion ( $\mathrm{Ng}$ et al., 2013), and E. coli during intestinal inflammation (Huang et al., 2015). Humans readily synthesize the Neu5Ac type of sialic acid and have dedicated membrane transporters to deploy it onto their surface. These share little homology to the bacterial transporters (North et al., 2017) so inhibitors to the bacterial transporters may not be toxic.

Recently, we determined the high-resolution outward-facing, and open, substrate-bound structure of the SiaT sialic acid transporter from Proteus mirabilis (PmSiaT) (Wahlgren et al., 2018). SiaT transporters belong to the SSS family. PmSiaT adopts the LeuT-fold with Neu5Ac bound near the center of the protein, and two $\mathrm{Na}^{+}$ions for transport.

This work investigates the SiaT sodium sialic acid symporter from $S$. aureus (SaSiaT). We demonstrate that SaSiaT can be purified and stably occupies a monomeric oligomeric state. We characterize the functionality of SaSiaT with two different sialic acids, and the kinetics of sialic acid membrane transport.

\section{MATERIALS AND METHODS}

\section{Molecular Biology Techniques}

The S. aureus (strain RF122) siaT (Accession AJ938182.1) gene was codon optimized for E. coli (GeneArt, ThermoFischer Scientific; Supplementary Figure 1). For purification of recombinant protein and functional studies, siaT was amplified by PCR using $S a \_s i a T-F 1$ and $S a \_s i a T-R 1$ oligonucleotides (Supplementary Table 1) and cloned into the pWarf(-) (Hsieh et al., 2010) vector using the In-Fusion HD Cloning Kit (Clontech). The pWarf(-) vector carries a C-terminal human rhinovirus $3 \mathrm{C}$ protease (HRV3C) cleavage site followed by a green fluorescence protein (GFP)-tag and an $8 \times$ histidine (His)-tag. The amplified fragment was cloned into pWarf(-) digested with the BamHI $\left(3^{\prime}\right)$ and XhoI $\left(5^{\prime}\right)$ restriction enzymes to generate pWarf(-)Sa_siaT with kanamycin resistance. This was transformed into Stellar ${ }^{\mathrm{TM}}$ Competent Cells (Clontech), purified using the DNA-Spin ${ }^{\mathrm{TM}}$ Plasmid DNA Purification Kit (iNtRon Biotechnology), and verified by DNA sequencing (Eurofins).

For bacterial growth experiments, siaT was amplified by PCR using $S a \_s i a T-F 2$ and $S a \_s i a T-R 2$ oligonucleotides (Supplementary Table 1) and cloned into the low-copy vector pJ422-01 also using the In-Fusion HD Cloning Kit. The amplified fragment was cloned into pJ422-01 digested with the EcoR1 $\left(3^{\prime}\right)$ and Nde1 $\left(5^{\prime}\right)$ restriction enzymes to generate pJ422$01 \mathrm{Sa}$ siaT with Zeocin ${ }^{\mathrm{TM}}$ resistance. This was transformed into Stellar ${ }^{\mathrm{TM}}$ Competent Cells (Clontech), purified using the DNASpin $^{\mathrm{TM}}$ Plasmid DNA Purification Kit (iNtRon Biotechnology), and verified by DNA sequencing (Genetic Analysis Service, University of Otago). The pJ422-01Sa_siaT plasmid was subsequently transformed into the E. coli JW3193 $\Delta$ nanT strain 
[NBRP (NIG, Japan): E. coli] (Baba et al., 2006) generating the complementation strain E. coli JW3193 $\Delta$ nanT-siaT.

\section{Protein Production and Purification}

The pWarf(-)Sa_siaT plasmid was transformed into E. coli Lemo21(DE3) and grown in terrific broth media supplemented with kanamycin $(50 \mu \mathrm{g} / \mathrm{mL})$, chloramphenicol $(34 \mu \mathrm{g} / \mathrm{mL})$, Lrhamnose $(100 \mu \mathrm{M})$, and induced with $0.4 \mathrm{mM}$ isopropyl $\beta$ D-1-thiogalactopyranoside (IPTG) at $26^{\circ} \mathrm{C}$ overnight, with shaking at $180 \mathrm{rpm}$. For isothermal titration calorimetry and proteoliposome measurements, the protein was expressed in PASM-5052 auto-induction media (Lee et al., 2014). Cells were solubilized in phosphate-buffered saline (PBS), supplemented with cOmplete ${ }^{\mathrm{TM}}$ EDTA free protease inhibitor tablets (Roche), lysozyme $(0.5 \mathrm{mg} / \mathrm{mL})$, DNaseI $(5 \mu \mathrm{g} / \mathrm{mL}), \mathrm{MgCl}_{2}(2 \mathrm{mM})$ and lysed by sonication using a Hielscher UP200S Ultrasonic Processor at $70 \%$ amplitude in cycles of $0.5 \mathrm{~s}$ on, $0.5 \mathrm{~s}$ off, for $30 \mathrm{~min}$. Cell debris was pelleted by centrifugation at $24,000 \mathrm{~g}$, for $25 \mathrm{~min}$, at $4^{\circ} \mathrm{C}$ and the cell membranes were collected by ultracentrifugation at $230,000 \mathrm{~g}$, for $2 \mathrm{~h}$, at $4^{\circ} \mathrm{C}$ and stored at $-80^{\circ} \mathrm{C}$ until further use. Cell membranes were solubilized in $2 \%(\mathrm{w} / \mathrm{v}) \mathrm{n}$-dodecyl- $\beta$-D-maltoside (DDM) for $2 \mathrm{~h}$ at $4^{\circ} \mathrm{C}$ and unsolublized material was removed by ultracentrifugation at $150,000 \mathrm{~g}$. The protein was first purified using immobilized metal affinity chromatography; the supernatant was loaded onto a $5 \mathrm{~mL}$ HisTrap HP column (GE Healthcare) equilibrated with Buffer A (70 mM Tris- $\mathrm{HCl}, \mathrm{pH} 8.0,150 \mathrm{mM} \mathrm{NaCl}, 20 \mathrm{mM}$ imidazole, $6 \%$ glycerol, $5 \mathrm{mM} \beta$-mercaptoethanol, and $0.0174 \%$ (w/v) DDM). The column was washed with Buffer A, followed by a $10 \%$ wash with Buffer B (Buffer A with $500 \mathrm{mM}$ imidazole) and protein was eluted using 50\% Buffer B. Protein was concentrated and simultaneously exchanged into Buffer C (50 mM Tris- $\mathrm{HCl}$, pH 8.0, $150 \mathrm{mM} \mathrm{NaCl}, 0.0174 \%$ (w/v) DDM). For analytical ultracentrifugation experiments, Buffer C contained $0.174 \%$ DDM. The GFP-tag was cleaved with HRV3C protease in a 1:12.5 mass ratio (HRV3C:SaSiaT) at $4^{\circ} \mathrm{C}$ for $18 \mathrm{~h}$. Size exclusion chromatography was performed as a final purification step using a HiLoad 16/60 Superdex 200 column in Buffer C. Protein concentration was determined using a NanoDrop 1000 spectrophotometer at $280 \mathrm{~nm}$, using an extinction coefficient of $75,750 \mathrm{M}^{-1} \mathrm{~cm}^{-1}$, and a molecular weight of $56.7 \mathrm{kDa}$ following HRV3C cleavage of the GFP-tag.

\section{Analytical Ultracentrifugation}

Sedimentation velocity experiments were performed in a Beckman Coulter Model XL-I analytical ultracentrifuge equipped with UV/Vis scanning optics. Reference buffer solution $(50 \mathrm{mM}$ Tris- $\mathrm{HCl}, 150 \mathrm{mM} \mathrm{NaCl}, \mathrm{pH}$ 8.0) and sample solutions (including reference buffer solution with $0.174 \% \mathrm{DDM}$, and SaSiaT at four concentrations: $0.6,0.4,0.2$, and $0.1 \mathrm{mg} \mathrm{mL}^{-1}$ ) were loaded into $12 \mathrm{~mm}$ double-sector cells with standard Epon 2-channel centerpieces and quartz windows. Cells were mounted in an eight hole An-50 Ti rotor and centrifuged at 50,000 rpm at $12^{\circ} \mathrm{C}$. Interference and absorbance measurements at a wavelength of $280 \mathrm{~nm}$ were recorded over a radial position range of 5.8 to $7.3 \mathrm{~cm}$ within the cell, with measurements taken at sediment boundary intervals of $0.003 \mathrm{~cm}$. The partial specific volume of
SaSiaT was calculated using SEDNTERP (Laue et al., 1992) and buffer density and buffer viscosity were experimentally measured with an Anton Paar DMA4100M density meter and Anton Paar Lovis $2000 \mathrm{ME}$ microviscometer, respectively. The van HoldeWeischet and 2DSA-Monte Carlo analyses were performed using UltraScan III (Demeler and van Holde, 2004; Brookes and Demeler, 2007; Demeler and Brookes, 2007; Demeler, 2010).

\section{Bacterial Growth Experiment}

E. coli strains JW3193 $\Delta$ nanT, JW3193 $\Delta$ nanT_siaT, and E. coli BW25113, which served as a wild type control, were grown $\left(37^{\circ} \mathrm{C}, 250 \mathrm{rpm}\right)$ overnight in low salt Luria-Bertani (LB) media. For $\Delta n a n T \_s i a T$, the LB media was supplemented with Zeocin $^{\mathrm{TM}}(25 \mu \mathrm{g} / \mathrm{mL})$. Overnight cultures were diluted to an $\mathrm{OD}_{600}$ of 0.05 and further grown $\left(37^{\circ} \mathrm{C}, 250 \mathrm{rpm}\right)$ in low salt LB media supplemented with $1 \mathrm{mM}$ IPTG until they reached mid-logarithmic phase $\left(\mathrm{OD}_{600}\right.$ of 0.35$)$. Bacterial cultures were harvested by centrifugation $\left(6,000 \mathrm{rpm}, 10 \mathrm{~min}, 4^{\circ} \mathrm{C}\right)$, washed three times in $\mathrm{M} 9$ minimal media and diluted to an $\mathrm{OD}_{600}$ of 0.5. Cultures $(20 \mu \mathrm{L})$ were added to a Costar Flat Bottom 96 well plate with lid containing M9 minimal media (180 $\mu \mathrm{L})$ supplemented with Zeocin ${ }^{\mathrm{TM}}(25 \mu \mathrm{g} / \mathrm{mL})$, IPTG $(1 \mathrm{mM})$, thiamin hydrochloride $(7 \mu \mathrm{M})$ and either $N$-acetylneuraminic acid (Neu5Ac, $4 \mathrm{mg} / \mathrm{mL}, 12.9 \mathrm{mM}$ ), $N$-glycolylneuraminic acid (Neu5Gc, $4 \mathrm{mg} / \mathrm{mL}, 12.3 \mathrm{mM}$ ) or glucose $(0.4 \%)$ as the sole carbon source. In addition, bacterial growth was monitored in M9 minimal media without any carbon source. Growth at $37^{\circ} \mathrm{C}$, with shaking at $250 \mathrm{rpm}$, was recorded at $600 \mathrm{~nm}$ every $10 \mathrm{~min}$ using the FLUOstar Omega microplate reader (BMG labtech). Growth curves represent the mean of four measurements \pm standard error of the mean, or three measurements \pm standard error of the mean for the control experiments.

\section{Microscale Thermophoresis}

The binding affinities for two sialic acids and purified SaSiaT were determined using microscale thermophoresis. Experiments were performed on a Monolith NT.LabelFree instrument (NanoTemper Technologies) (Wienken et al., 2010; Soares da Costa et al., 2016; Stifter et al., 2018). Purified SaSiaT was diluted to $2 \mu \mathrm{M}$ in PBS buffer supplemented with $0.0174 \%$ (w/v) DDM, and incubated with Neu5Ac (from $0.3 \mu \mathrm{M}$ to $10 \mathrm{mM})$, and Neu5Gc $(0.08 \mu \mathrm{M}$ to $2.5 \mathrm{mM})$, for $5 \mathrm{~min}$ prior to taking measurements. The samples were loaded into Monolith NT Standard Treated Capillaries (NanoTemper Technologies). Microscale thermophoresis measurements were carried out at $25^{\circ} \mathrm{C}$ using $20 \%$ LED power, and $20 \%$ microscale thermophoresis infrared laser power. The dissociation constants $\left(K_{d}\right)$ were determined using the mass action equation via the NT Analysis software version 1.5.41 (NanoTemper Technologies), using the signal from Thermophoresis + T-jump for triplicate experiments.

\section{Isothermal Calorimetry}

Purified SaSiaT was concentrated to a final concentration of 170$240 \mu \mathrm{M}$ using membrane ultrafiltration with a molecular weight 
cutoff of $50 \mathrm{kDa}$. The flow through was used to dilute $100 \mathrm{mM}$ stock solutions of sialic acid to concentrations of 2.5-4 mM for Neu5Ac and 2-2.4 mM for Neu5Gc. Protein sample (206 $\mu \mathrm{L})$ was loaded into the sample cell, and $70 \mu \mathrm{L}$ of the respective sialic acid was loaded into the injection syringe. The system was equilibrated to $25^{\circ} \mathrm{C}$ with a stirring speed of $750 \mathrm{rpm}$. Titration curves were initiated by a $1 \mu \mathrm{L}$ injection followed by $4 \mu \mathrm{L}$ injections every $180 \mathrm{~s}$. Background corrections were obtained by injection of sialic acids into buffer and buffer into protein with the same parameters. Biological triplicate experiments were analyzed using ORIGIN 7 with the first injection excluded. The curves were fitted into a single-site binding isotherm and $K_{d}$ values were determined. Measurements were made using a Micro-200 Isothermal titration calorimeter or a PEAQ Isothermal titration calorimeter (MicroCal, Malvern).

\section{Sequence Alignment and Homology Modeling of SiaT in an Outward-Facing Open Conformation}

Multiple protein sequence alignment was performed between SaSiaT and additional SiaT from various bacterial species, as described elsewhere (Wahlgren et al., 2018). This was used to compare conservation of the Neu5Ac binding site between organisms. A second multiple protein sequence alignment was performed between SiaT from 19 strains of S. aureus, to compare conservation of the Neu5Ac binding site between different isolates. These include S. aureus RF122, S. aureus ED133, S. aureus NR153, S. aureus XQ, S. aureus 93b_S9, S. aureus CFSAN007883, S. aureus HZW450, S. aureus MS4, S. aureus SA268, S. aureus SA40, S. aureus SA957, S. aureus M013, S. aureus FDA209P, S. aureus FDAARGOS_43, S. aureus NRS271, S. aureus MW2, S. aureus NRS143, S. aureus USA400-0051, S. aureus EDCC5464. Alignments were generated using ClustalW (Larkin et al., 2007).

The outward-facing open conformation of SaSiaT was modeled on the outward-facing open structure of SiaT from P. mirabilis (PmSiaT). These transporters share $\sim 41 \%$ sequence identity. To build a homology model, an alignment between the two protein sequences was first generated using a global sequence alignment with EMBOSS stretcher (Myers and Miller, 1988). MEDELLER (Kelm et al., 2010) was used to create the model of $\mathrm{SaSiaT}$ in the outward-facing open conformation using PmSiaT (pdb entry 5nv9) as a template structure. Next, GROMACS 5.1.2 (Abraham et al., 2015) was used for energy minimization of the SaSiaT homology model, using the GROMOS 54A7 force field. The resulting structure was superimposed onto the PmSiaT structure with Neu5Ac bound using a Structural Alignment of Multiple Proteins (STAMP) structure-based sequence alignment in VMD MultiSeq. The structure was manually edited using COOT (without further energy minimization) to remove a clash between the sidechain of Tyr79 and the Neu5Ac. The sidechain was rotated to overlay with that of the corresponding residue (Phe78) in the template PmSiaT structure. Other residues in the substrate binding site of the homology model were rotated to better represent the conformations found in the substrate bound PmSiaT template.

\section{Proteoliposome Assays}

Purified SaSiaT was reconstituted into proteoliposomes using a protocol previously optimized for PmSiaT, with some modifications (Wahlgren et al., 2018). Briefly, $5 \mu \mathrm{g}$ of protein was mixed with $120 \mu \mathrm{L} 10 \% \mathrm{C}_{12} \mathrm{E}_{8}, 100 \mu \mathrm{L}$ of $10 \%$ egg yolk phospholipids ( $\mathrm{w} / \mathrm{v}$, sonicated as previously described to form liposomes, Scalise et al., 2014). Next, $20 \mathrm{mM}$ of $\mathrm{K}^{+}$-gluconate buffered by $20 \mathrm{mM}$ Tris- $\mathrm{HCl}$, pH 7.0 was added to create a final volume of $700 \mu \mathrm{L}$. The mixture was incubated with $0.5 \mathrm{~g}$ Amberlite XAD-4 resin under rotatory stirring (1,200 rev/min) at $25^{\circ} \mathrm{C}$ for $40 \mathrm{~min}$ (Scalise et al., 2015). After reconstitution, $600 \mu \mathrm{L}$ of proteoliposomes were loaded onto a Sephadex G75 column $(0.7 \mathrm{~cm}$ diameter $\times 15 \mathrm{~cm}$ height $)$ pre-equilibrated with $20 \mathrm{mM}$ Tris- $\mathrm{HCl}, \mathrm{pH} 7.0,40 \mathrm{mM}$ sucrose to balance internal osmolarity. To generate a $\mathrm{K}^{+}$diffusion potential, valinomycin $(0.75 \mu \mathrm{g} / \mathrm{mg}$ phospholipid) prepared in ethanol was added to the proteoliposomes following Sephadex G-75 column chromatography, as previously described (Scalise et al., 2014; Wahlgren et al., 2018). As a control, ethanol was added to proteoliposomes, which did not exert any effect on the transport activity. After $10 \mathrm{~s}$ of incubation with valinomycin/ethanol, transport was started by adding $50 \mu \mathrm{M}\left[{ }^{3} \mathrm{H}\right]$-Neu5Ac to the proteoliposomes in the presence of $25 \mathrm{mM} \mathrm{NaCl}$. The initial rate of transport was measured by stopping the reaction after $5 \mathrm{~min}$, i.e., within the initial linear range of $\left[{ }^{3} \mathrm{H}\right]-\mathrm{Neu} 5 \mathrm{Ac}$ uptake into the proteoliposomes. Transport was terminated once $\left[{ }^{3} \mathrm{H}\right]-$ Neu5Ac was removed by loading each proteoliposome sample $(100 \mu \mathrm{L})$ on a Sephadex G-75 column $(0.6 \mathrm{~cm}$ diameter $\times 8 \mathrm{~cm}$ height). Proteoliposomes were eluted with $1 \mathrm{~mL} 50 \mathrm{mM} \mathrm{NaCl}$ and collected in $4 \mathrm{~mL}$ of scintillation mixture, vortexed and counted. Radioactivity uptake in liposome controls (without incorporated protein) was negligible with respect to transport data. Uptake data were fitted in a first-order rate equation for time course plots, and kinetic data were fitted with a Michaelis-Menten or Hill equations. Non-linear fitting analysis was performed by Grafit software (version 5.0.13). To measure the specific activity of SaSiaT, the amount of protein was estimated by NanoDrop. All measurements are presented as means \pm SD from three independent experiments.

\section{RESULTS AND DISCUSSION}

\section{Expression of SaSiaT Rescues an E. Coli Strain That Lacks its Endogenous Sialic Acid Transporter}

To demonstrate sialic acid transport by $\mathrm{SaSiaT}$, we first showed that SaSiaT rescues the growth of an E. coli strain lacking the endogenous NanT sialic acid transporter $(\Delta n a n T)$ when grown on Neu5Ac or Neu5Gc (Figure 1). The Neu5Ac and Neu5Gc differ by the addition of a hydroxyl at the C11 methyl of the $N$-acetyl group in Neu5Gc (Figure 1A). While E. coli JW3193 $\Delta$ nanT grows in M9 minimal media supplemented with glucose (Table 1), it is not able to utilize Neu5Ac or Neu5Gc as the sole carbon source (Figure 1B, data in green). After complementation of E. coli JW3193 $\Delta n a n T$ with pJ422-01Sa_siaT (to produce E. coli JW3193 
A

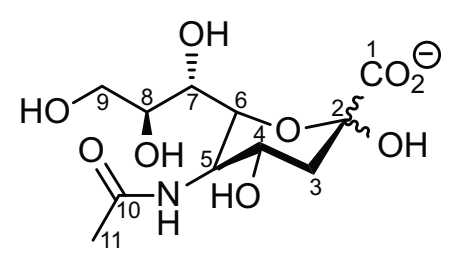

$\mathrm{N}$-acetylneuraminic acid (Neu5Ac)

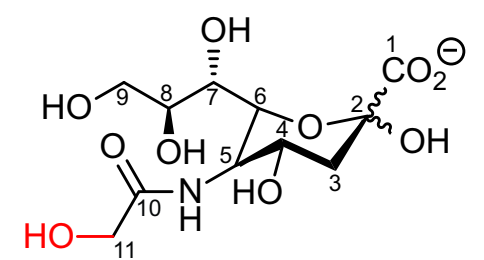

$\mathrm{N}$-glycolylneuraminic acid (Neu5Gc)

B
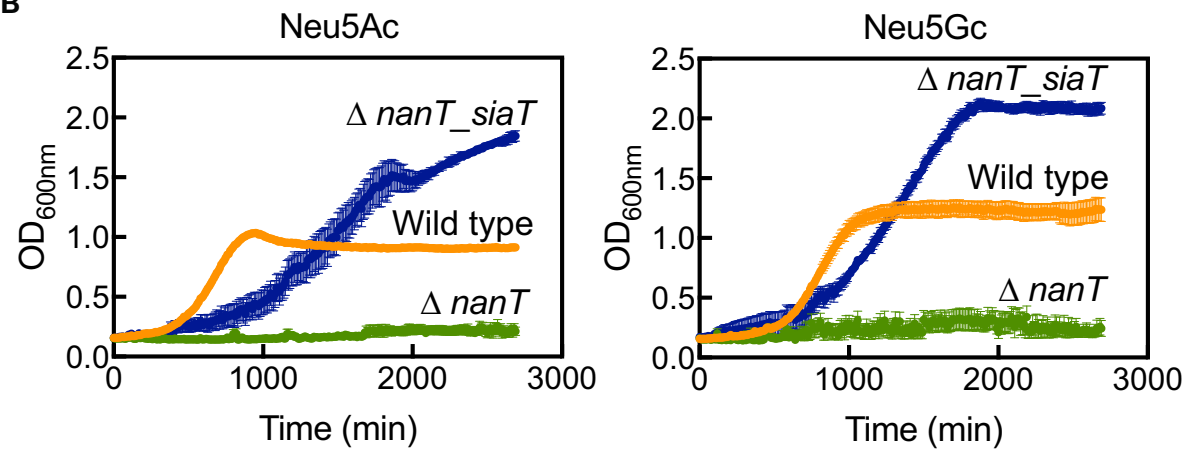

FIGURE 1 | Bacterial growth experiments demonstrate SaSiaT function. (A) The chemical structures of Neu5Ac and Neu5Gc. (B) Growth of E. coli wild type (orange), $\Delta$ nant (green), and its complemented derivative $\Delta$ nant_siaT (blue) on Neu5Ac and Neu5Gc. While $\Delta$ nanT is unable to utilize Neu5Ac and Neu5Gc, SaSiaT is able to rescue the growth of $\triangle$ nanT on both Neu5Ac and Neu5Gc sialic acids as the sole carbon source.

TABLE 1 | Growth rates of the E. coli wild type, $\Delta$ nanT and $\Delta$ nanT_siaT in M9 minimal media containing different carbon sources.

\begin{tabular}{|c|c|c|c|c|}
\hline Strain & Neu5Ac & Neu5Gc & Glucose & No carbon source \\
\hline Wild type & $3.56(0.04) \times 10^{-3}$ & $3.3(0.1) \times 10^{-3}$ & $5.5(0.2) \times 10^{-3}$ & - \\
\hline$\Delta$ nant & - & - & $4.3(0.5) \times 10^{-3}$ & - \\
\hline$\Delta$ nant_siaT & $1.3(0.3) \times 10^{-3}$ & $1.9(0.05) \times 10^{-3}$ & $3.86(0.05) \times 10^{-3}$ & - \\
\hline
\end{tabular}

Values represent the growth rate/min. A dash (-) indicates no growth. Values in brackets represent the standard error of measurement, where $n=4$.

$\left.\Delta n a n T \_s i a T\right)$, the ability to grow on both sialic acids is restored (Figure 1B, data in blue). Notably, the growth rate of $\Delta$ nanT_siaT is faster when grown in M9 minimal media containing Neu5Gc as the sole carbon source, as opposed to Neu5Ac (Table 1). This could reflect more efficient transport of Neu5Gc, due to a higher affinity for Neu5Gc compared to Neu5Ac.

Curiously, E. coli $\Delta n a n T \_s i a T$ reached higher final optical density compared to wild type E. coli BW25113 (Figure 1B, data in blue compared to data in orange). This was unexpected, perhaps suggesting that the native E. coli NanT may be regulated in some way, thereby limiting sialic acid uptake. E. coli $\Delta n a n T \_s i a T$ exhibits an extended lag-phase compared to wild type E. coli BW25113. Since SiaT expression was pre-induced with IPTG (see section Materials and Methods), it is likely that the growth lag is due to an increased metabolic burden caused by the overexpression of SiaT itself. This also explains the reduced growth rates of $\Delta n a n T \_s i a T$ compared to the wild type (Table 1).
In short, we demonstrate that the SaSiaT is functional in vivo and observe a preference for Neu5Gc in terms of maximum growth rate.

\section{Recombinant SaSiaT Is Stably Purified as a Single Species}

SaSiaT was successfully overexpressed, solubilized, and purified to homogeneity in buffer containing DDM detergent. The profile from size exclusion chromatography (Figure 2A) shows a dominant peak at $\sim 55 \mathrm{~mL}$, with a shoulder to the left that is consistent with a small amount of a larger component, possibly an aggregate. Analytical ultracentrifugation studies at protein concentrations ranging from 0.1 to $0.6 \mathrm{mg} / \mathrm{mL}$ were used to assess the stability and oligomeric state of purified recombinant SaSiaT prior to functional studies. Analyses of absorbance data from analytical ultracentrifugation experiments, using van HoldeWeischet sedimentation coefficient distributions (Figure 2B), reveal a largely monodisperse solution with a major component at $\sim 8 \mathrm{~S}$. However, as evidenced by the shift of the distribution 

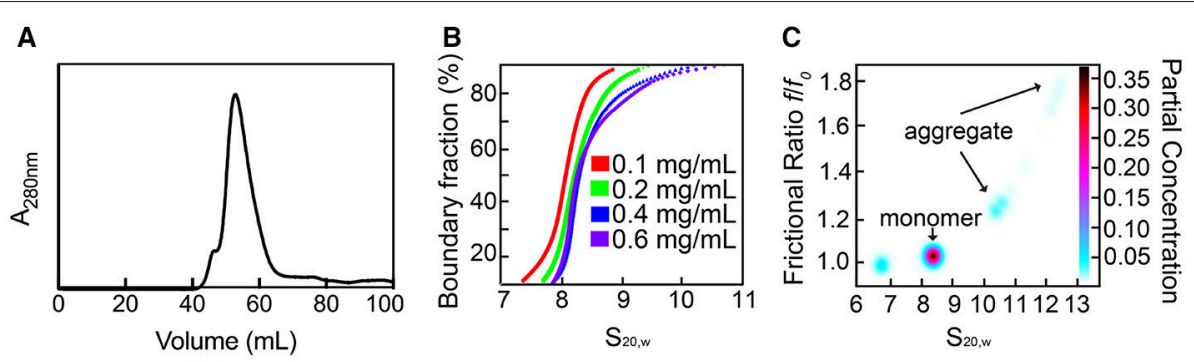

FIGURE 2 | Recombinant SaSiaT can be stably purified and occupies a predominantly single oligomeric state. (A) Size-exclusion chromatography trace of SaSiaT at its final purification step. To the left of a main dominant peak there is a small shoulder, which may represent aggregate. (B) van Holde-Weischet sedimentation coefficient distributions show a dominant component at $\sim 8 \mathrm{~S}$. (C) 2DSA-Monte Carlo analysis of sedimentation velocity data of SaSiaT at $0.6 \mathrm{mg} / \mathrm{mL}$, as implemented by UltraScan III, shows a main peak comprising $70 \%$ of the signal.

to the right, the van Holde-Weischet analysis also suggests that some protein may be aggregated. 2DSA-Monte Carlo analysis (Figure 2C) determines a major component at $8.2 \mathrm{~S}$ with a frictional ratio of 1.05 , which is consistent with a molecular weight of $160 \mathrm{kDa}$ (assuming a mass averaged v-bar of 0.76 for the detergent:protein complex). This is consistent with a SaSiaT monomer surrounded by $\sim 200$ DDM molecules. This monomeric species represents $\sim 70 \%$ of the total sample. The 2DSA-Monte Carlo analysis also shows a series of larger components ranging from 10.5 to $13.0 \mathrm{~S}$ with an increasing frictional ratio, that represent a small amount of aggregate. Overall, our studies demonstrate that SaSiaT can be stably expressed and purified using the detergent DDM. Furthermore, when solubilized in DDM, it is largely a monomer that associates with $\sim 200$ DDM molecules in solution.

\section{Binding Studies Demonstrate That SaSiaT Has an Altered Specificity}

Given the increased growth rate observed when grown on Neu5Gc compared to Neu5Ac, the binding affinity of Neu5Ac and Neu5Gc to recombinant SaSiaT was determined using microscale thermophoresis (Figure 3A) and isothermal titration calorimetry (Figure 3B). By microscale thermophoresis, SaSiaT has a considerably higher affinity for the Neu $5 \mathrm{Gc}$ sialic acid $\left(K_{d}\right.$ $=39 \pm 4 \mu \mathrm{M})$ compared to the Neu5Ac sialic acid $\left(K_{d}=113\right.$ $\pm 6 \mu \mathrm{M})$. Consistent with this, isothermal titration calorimetry experiments confirmed that SaSiaT has a higher affinity for $\operatorname{Neu} 5 \mathrm{Gc}\left(K_{d}=18 \pm 2 \mu \mathrm{M}\right)$ than Neu5Ac $\left(K_{d}=106 \pm 2 \mu \mathrm{M}\right)$. Interestingly, $P m S i a T$ is the opposite, and displays a more similar, but higher binding affinity for Neu5Ac $\left(K_{d}^{\mathrm{Neu} 5 \mathrm{Ac}}=58 \pm 1 \mu \mathrm{M}\right)$ compared to Neu5Gc $\left(K_{d}{ }^{\mathrm{Neu} 5 \mathrm{Gc}}=85 \pm 2 \mu \mathrm{M}\right)$ using microscale thermophoresis (Wahlgren et al., 2018). Thus, SaSiaT reveals different substrate specificity compared to PmSiaT.

\section{Three Substitutions in the Active Site of SaSiaT may Explain Altered Substrate Specificity}

To reconcile at the molecular level the observed difference in substrate specificity, and ultimately, the difference between
SaSiaT and PmSiaT, amino acid sequence analyses and homology modeling were used to examine the differences in the active site.

The Neu5Ac binding site, as determined in PmSiaT (pdb entry $5 \mathrm{nv} 9$ ), is conserved among SiaT transporters from a number of bacterial species (Figure 4A) (Wahlgren et al., 2018). When comparing the sequences between SaSiaT and PmSiaT, there are three substitutions in SaSiaT (PmSiaT-Gln82 to SaSiaT-Asn83, PmSiaT-Phe78 to SaSiaT-Tyr79, and PmSiaT-Phe243 to SaSiaTAsn244, Figure 4A) that we predict are involved in substrate binding. These residues are highly conserved among $S$. aureus isolates (Supplementary Figure 2); they are, therefore, not specific to any particular isolate of $S$. aureus.

To map these substitutions within the putative active site, a homology model of SaSiaT was built based upon the outwardfacing open structure of PmSiaT (pdb entry 5nv9) (Figure 4B). Superposition of the SaSiaT homology model with PmSiaT (r.m.s.d. $=0.172 \AA$ for $364 \alpha$-carbon atoms) demonstrates that the PmSiaT-Gln82 to SaSiaT-Asn83, PmSiaT-Phe243 to SaSiaTAsn244, and PmSiaT-Phe78 to SaSiaT-Tyr79 substitutions in SaSiaT may be responsible for the altered substrate specificity observed in the binding experiments, since they are close to the C11 methyl group of the $N$-acetyl moiety of Neu5Ac (Figure 4B), which is hydroxylated in Neu5Gc.

In the PmSiaT crystal structure, the side chain of Gln 82 (SaSiaT-Asn83) is in a position to form two hydrogen bonds with the hydroxyl group at $\mathrm{C} 9$ and the hydroxyl group at $\mathrm{C} 7$ of the Neu5Ac glycerol tail (Figure 4C) (Wahlgren et al., 2018). In SaSiaT, the side chain of the Asn83 substitution is shorter than a Gln, which creates more space in the substrate-binding cavity. SaSiaT-Asn83 may be in a position to bond the additional C11 hydroxyl of Neu5Gc (Figure 4C), but only if the C11 hydroxyl points toward the glycerol tail. Like PmSiaT-Gln82, SaSiaTAsn83 is still in position to hydrogen bond the hydroxyl group at $\mathrm{C} 9$ of the glycerol tail, albeit with a longer hydrogen bond length.

The C10 carbonyl of the $N$-acetyl moiety in Neu5Ac is coordinated by the amide from the side chain of Gln 82 through a water molecule, while the methyl group of the $\mathrm{N}$-acetyl moiety of Neu5Ac is facing toward a hydrophobic patch formed by Phe78 and Phe243 (Figure 4C) (Wahlgren et al., 2018). In SaSiaT, Tyr79, and Asn244 replace the equivalent positions of 

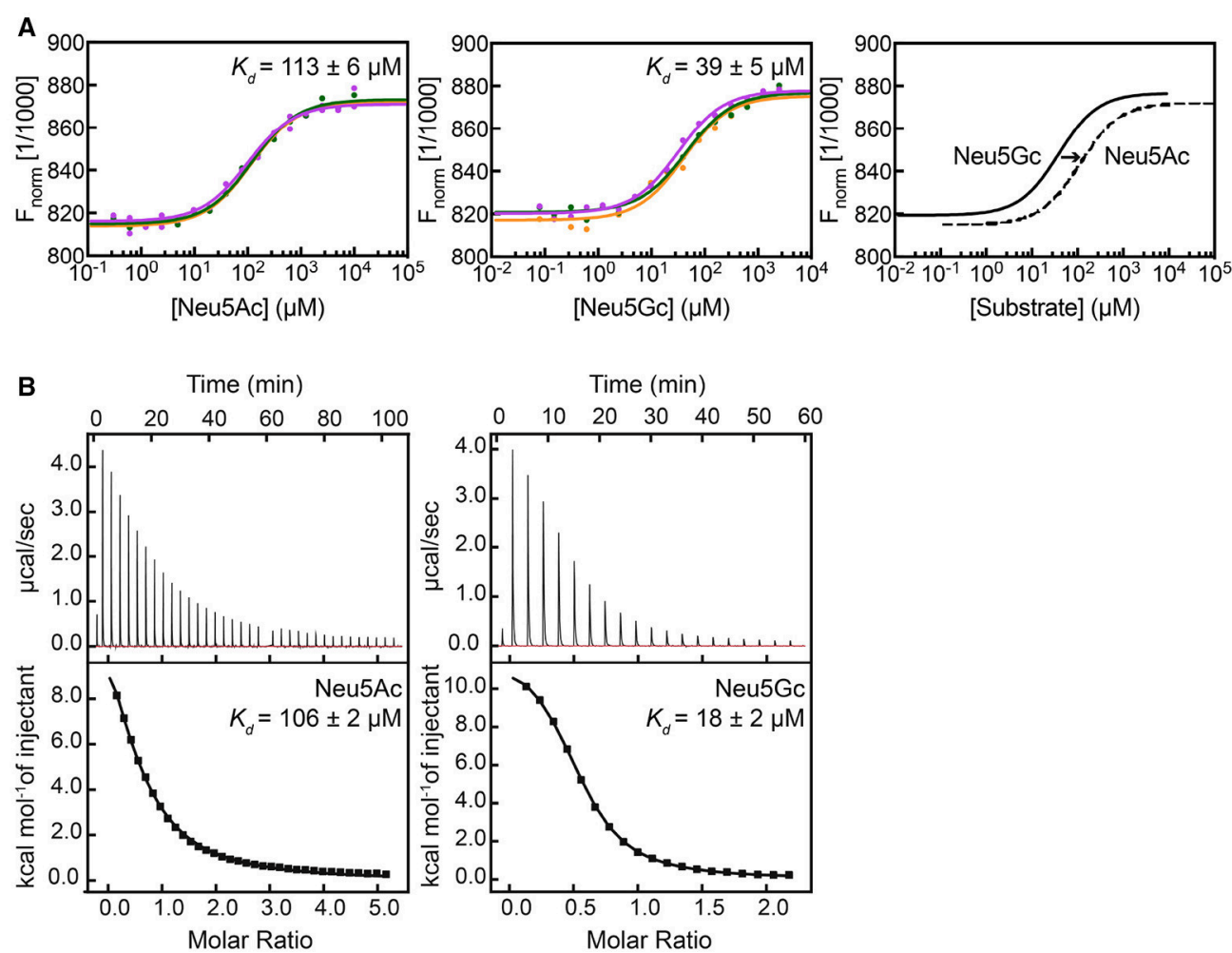

FIGURE 3 | Orthogonal binding experiments demonstrate substrate ambiguity. (A) Microscale thermophoresis binding assay to measure the affinity of SaSiaT for Neu5Ac and Neu5Gc sialic acids. Raw data are shown with the fit for three independent experiments with Neu5Ac (left) and Neu5Gc (middle). The $K_{d}$ values are reported as the mean \pm uncertainty in the mean of the fit using the signal from Thermophoresis + T-jump, from triplicate experiments, where $n=1$ (we define $n$ as the number of different recombinant protein preparations, which we view as equivalent to biological replicates). The $K_{d}$ and associated error of each fit is given in Supplementary Table 2. The shift in $K_{d}$ between both sialic acids is shown (right). SaSiaT has a tighter affinity for Neu5Gc than Neu5Ac. (B) Representative isothermal titration calorimetry raw data (top panel) and binding isotherm (bottom panel) of one isothermal titration calorimetry experiment obtained by successive titration of Neu5Ac (left) or Neu5Gc (right) with purified SaSiaT. The fit of a single binding site is shown in the bottom panels (black line). $K_{d}$ values are reported as the mean \pm SEM of the fit from three experiments using different protein preparations $(n=3)$.

PmSiaT-Phe78 and PmSiaT-Phe243 (Figure 4B). SaSiaT-Asn244 could facilitate a new interaction with the additional C11 hydroxyl group of Neu5Gc, and the side chain hydroxyl group of SaSiaT-Tyr79 could create a more hydrophilic environment in the vicinity, which may be important for Neu5Ac and Neu5Gc discrimination.

There are other examples where the preference for Neu5Gc is mediated by new interactions, via hydrogen bonds, with the extra hydroxyl group present in Neu5Gc. A similar preference for Neu5Gc over Neu5Ac has been reported for the subtilase cytotoxin (SubAB) produced by Shiga-toxigenic E. coli (Byres et al., 2008), and the porcine rotavirus (Yu et al., 2012), both of which bind to glycans terminating with sialic acids. The crystal structure of SubB-Neu5Gc complex (pdb entry 3dwa) shows that the C11 hydroxyl group of the glycolyl in Neu5Gc forms important hydrogen bonds with the side chain of a Tyr, and the main chain of a Met (Byres et al., 2008). The crystal structure of the porcine rotavirus strain CRW-8 spike protein domain VP8 (pdb entry 3tay) has similar interactions between the glycolyl of Neu5Gc with the side chain of a Thr, and the main chain of a Tyr (Yu et al., 2012). Mutation of these residues results in a significant loss of activity. Similarly, the VP1 capsid protein from human polyomavirus 9 (HPyV9) has a preference for Neu5Gc over Neu5Ac (Khan et al., 2014). However, the VP1 capsid protein from a closely related homolog, monkey-derived simian B-lymphotropic polyomavirus (LPyV), has no such preference (Khan et al., 2014). Again, the preference for Neu5Gc is acquired by specific hydrogen bonds with the glycolyl of Neu5Gc, which LPyV cannot form.

In conclusion, we suggest that the altered specificity of SaSiaT for Neu5Gc over Neu5Ac, compared to PmSiaT, may be afforded by Asn substitutions at the 83 and 244 positions, and a Tyr substitution at position 78 , in the substrate-binding site of SaSiaT.

\section{Proteoliposome Assays Delineate the Kinetics of Sialic Acid Membrane Transport}

To demonstrate the ability of purified recombinant $\mathrm{SaSiaT}$ to not only bind sialic acids, but to also transport them across a lipid membrane, we reconstituted the protein into proteoliposomes using native $E$. coli lipids and measured time dependent 


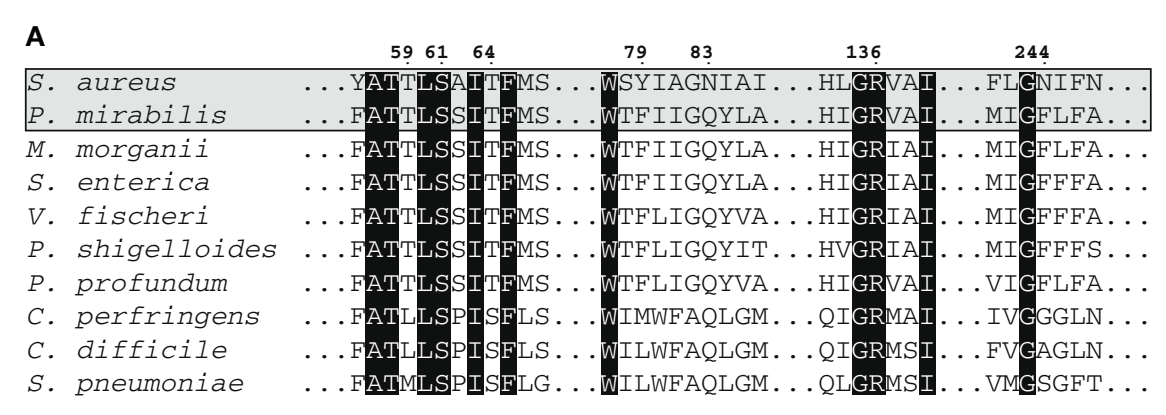

\section{B}
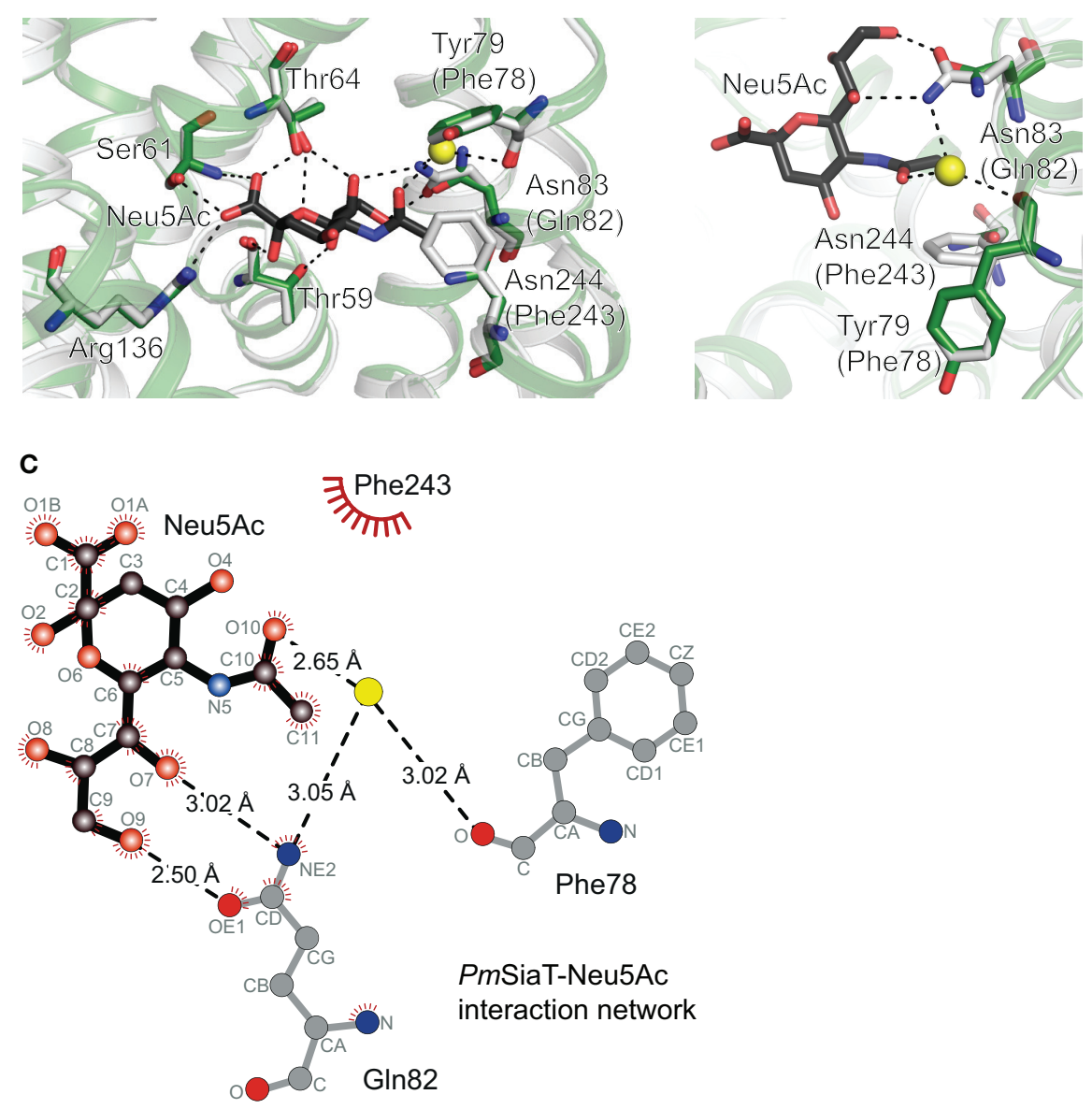

FIGURE 4 | Sequence alignment and homology modeling probe substrate ambiguity. (A) Amino acid sequence alignment of SaSiaT with SiaT transporters from eight additional bacterial species (Wahlgren et al., 2018). SiaT transporters from S. aureus, P. mirabilis, Morganella morganii, S. enterica, Vibrio fischeri, Plesiomonas shigelloides, Photobacterium profundum, Clostridium perfringens, C. difficile, and Streptococcus pneumoniae are aligned. Important residues in the Neu5Ac binding site in PmSiaT (pdb entry 5nv9) are shown. Residues highlighted with black boxes are highly conserved, and important residues implicated in Neu5Ac binding in PmSiaT are numbered according to SaSiaT. (B) Superposition of the SaSiaT homology model (green) and PmSiaT (gray) with Neu5Ac bound (black). Residues are labeled according to SaSiaT, with PmSiaT in parentheses. A water molecule from PmSiaT is shown in yellow. PmSiaT coordinates are from pdb entry 5nv9. Black dashed lines depict hydrogen bonds, or a salt bridge with Arg136. On the right, the binding site has been rotated $90^{\circ}$ and the substituted residues are shown. (C) The PmSiaT-Neu5Ac interaction network (Wahlgren et al., 2018) with Gln82, Phe78, and Phe243 is represented as a Ligplot ${ }^{+}$diagram (Laskowski and Swindells, 2011) using PDB entry 5nv9. Hydrogen bonds (dashed lines), hydrophobic contacts (arcs with spokes), and an interacting water molecule (yellow) are shown.

uptake of $\left[{ }^{3} \mathrm{H}\right] \mathrm{Neu} 5 \mathrm{Ac}$ (Figure 5A). The transporter mediated a $\mathrm{Na}^{+}$-dependent uptake of $\left[{ }^{3} \mathrm{H}\right] \mathrm{Neu} 5 \mathrm{Ac}$, stimulated by an imposed membrane potential. Similar to PmSiaT (Wahlgren et al., 2018), in the presence of an imposed membrane potential, transport at equilibrium was almost doubled (185 $\pm 15 \mathrm{nmol} / \mathrm{min} / \mathrm{mg}$ ) compared with transport in the absence of an imposed membrane potential (95 $\pm 5 \mathrm{nmol} / \mathrm{min} / \mathrm{mg}$ ) (Figure 5A). Transport of $\left[{ }^{3} \mathrm{H}\right] \mathrm{Neu} 5 \mathrm{Ac}$ in liposomes without 


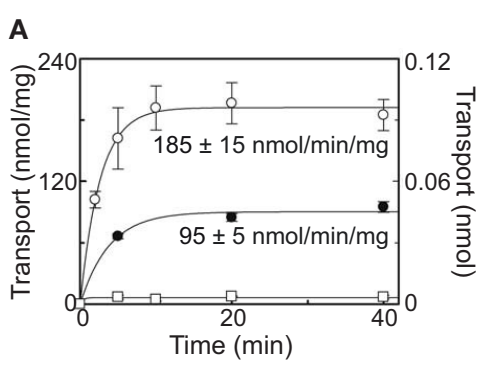

B

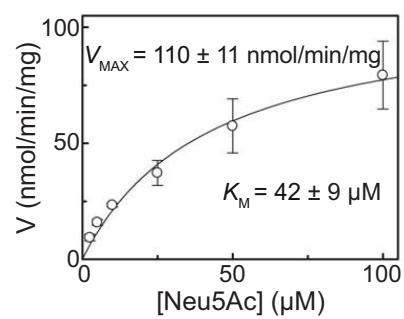

C

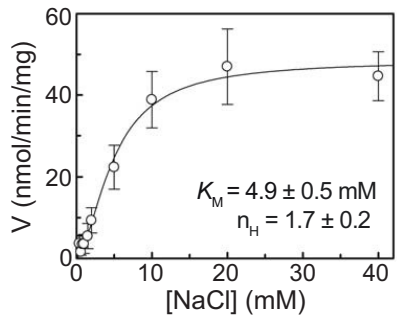

FIGURE 5 | Proteoliposome assays demonstrate the ability to transport sialic acid, which is dependent on Na+. (A) Proteoliposome transport was started by adding $50 \mu \mathrm{M}\left[{ }^{3} \mathrm{H}\right]-\mathrm{Neu} 5 \mathrm{Ac}$ together with $25 \mathrm{mM} \mathrm{NaCl}$ to proteoliposomes reconstituted with purified recombinant SaSiaT. In o, $\square$, valinomycin was added to facilitate $\mathrm{K}^{+}$ movement prior to transport. In $\bullet$, ethanol was added instead of valinomycin as a control. On the left Y-axis, specific transport activity is reported. In $\square$, transport was measured in empty liposomes, with transport in empty liposomes reported on the right $Y$-axis. Transport was stopped at indicated times by passing proteoliposomes through Sephadex-G75 columns. Data were fitted to the first-order rate equation. (B) The transport of [ $\left.{ }^{3} \mathrm{H}\right]-\mathrm{Neu} 5 \mathrm{Ac}$ over a range of concentrations in the presence of $25 \mathrm{mM} \mathrm{NaCl}$ was measured in proteoliposomes reconstituted with purified recombinant SaSiaT, with an imposed $\mathrm{K}^{+}$diffusion membrane potential, over 5 min. Data were fitted to the Michaelis-Menten equation. (C) The transport of $50 \mu \mathrm{M}\left[{ }^{3} \mathrm{H}\right]-\mathrm{Neu} 5 \mathrm{Ac}$ in the presence of $\mathrm{NaCl}$ over a range of concentrations was measured in proteoliposomes reconstituted with purified recombinant SaSiaT, with an imposed $\mathrm{K}^{+}$diffusion membrane potential, over 5 min. Data were fitted to the Hill equation. All data are presented as mean \pm SD from three independent experiments.

SaSiaT protein was negligible with respect to reconstituted SaSiaT proteoliposomes.

In optimal proteoliposome transport conditions with an imposed membrane potential, $\left[{ }^{3} \mathrm{H}\right] \mathrm{Neu} 5 \mathrm{Ac}$ is transported by SaSiaT with a $K_{\mathrm{M}}$ of $42 \pm 9 \mu \mathrm{M}$ and a $V_{\max }$ of $110 \pm 11$ $\mathrm{nmol} / \mathrm{min} / \mathrm{mg}$ (Figure 5B). This is lower (a higher binding affinity) than the measurements made using microscale thermophoresis and isothermal titration calorimetry, which may reflect the altered solubilization of the transporter in lipid, as opposed to detergent. The $K_{M}$ for $\left[{ }^{3} \mathrm{H}\right] \mathrm{Neu} 5 \mathrm{Ac}$ transport by SaSiaT is almost twice that of PmSiaT (Wahlgren et al., 2018). Consistent with the binding experiments, the molecular basis of this difference is likely that SaSiaT has a lower affinity for Neu5Ac compared to PmSiaT.

The kinetics of $\mathrm{Na}^{+}$transport by SaSiaT was measured in proteoliposomes, giving a $K_{\mathrm{M}}$ of $4.9 \pm 0.5 \mathrm{mM}$ (Figure 5C). As demonstrated by the cooperativity index calculated from the Hill plot, the transport stoichiometry is more than one for $\mathrm{Na}^{+}$. This is in the same order calculated for PmSiaT, which transport two $\mathrm{Na}^{+}$for every Neu5Ac (Wahlgren et al., 2018).

To conclude, proteoliposome experiments demonstrate that the recombinant SaSiaT is functional and able to transport $\mathrm{Neu} 5 \mathrm{Ac}$, that an electrogenic gradient drives transport, that the affinity for Neu5Ac is less than for PmSiaT, and that two $\mathrm{Na}^{+}$ ions are transported for every sialic acid.

\section{CONCLUSIONS}

Overall, we demonstrate that SaSiaT is a functional sialic acid transporter, with a considerably higher binding affinity for Neu5Gc over Neu5Ac. Compared to PmSiaT, in which Neu5Gc and Neu5Ac have similar binding affinities (Wahlgren et al., 2018), SaSiaT has altered substrate specificity. We propose that three residues unique to the SaSiaT substrate-binding site (Tyr79, Asn83, and Asn244) achieve a higher affinity to Neu5Gc. Like SubAB, the porcine rotavirus, and $\mathrm{HPyV} 9$, which also have a preference for Neu5Gc over Neu5Ac (Byres et al., 2008; Yu et al., 2012; Khan et al., 2014), specific hydrogen bonds with the C11 hydroxyl of Neu5Gc, and a hydrophilic environment in the vicinity of the Neu5Gc glycolyl chain, afford this specificity (Khan et al., 2014).

Although humans cannot synthesize Neu5Gc, they can acquire it from red meat and milk in the diet (Varki, 2010; Varki et al., 2011). Consequently, metabolic incorporation of Neu5Gc has been identified in the human gut epithelium and kidney vasculature (Tangvoranuntakul et al., 2003; Byres et al., 2008; Banda et al., 2012). Since some bacteria and viruses can discriminate between sialic acid variants (Byres et al., 2008; Yu et al., 2012; Khan et al., 2014; Stencel-Baerenwald et al., 2014), this could, in turn, influence their host or tissue range. It is possible that the ability of SaSiaT to bind Neu5Gc with higher affinity (compared to Neu5Ac) confers an advantage to S. aureus in specific niches.

Because a functional sialic acid transporter is essential for the uptake and utilization of sialic acid in a range of pathogenic bacteria, these transporters present a new avenue for drug design. The work presented here underpins the development of inhibitors that target SiaT transporters, and in particular, $S$. aureus.

\section{AUTHOR CONTRIBUTIONS}

$\mathrm{RF}$ and RD conceived the project. Cloning and expression trials of SaSiaT were carried out by ED and SR. Large-scale expression, membrane preparation, and protein purification were carried out by ED, WW, DR, and RN. Experiments for whole-cell functional analysis were designed by EC, ED, RN, and DR, and carried out by RN and DR. DR analyzed the results. Analytical ultracentrifugation experiments were performed and analyzed by SK. Proteoliposome assays were designed by MS, WW, RD, and CI. MS conducted and analyzed these experiments. Microscale thermophoresis experiments were carried out and analyzed by 
RN, TS, and MP. Isothermal titration calorimetry was performed and analyzed by WW. RN, WW, and JA carried out homology modeling and analysis of SaSiaT. RN, WW, DR, MS, SK, CI, JA, $\mathrm{RF}$, and $\mathrm{RD}$ wrote the manuscript, while all authors discussed the results and made manuscript revisions.

\section{ACKNOWLEDGMENTS}

$\mathrm{RD}$ acknowledges the following for funding support, in part: (1) the Marsden Fund Council from Government funding, managed by Royal Society Te Apārangi (contract UOC1506) and (2) the US Army Research Laboratory and US Army Research Office under grant number W911NF-11-1-0481. RF acknowledges the following for funding support: (1) the Swedish Research Council (2011-5790), (2) the Swedish Research Council Formas (20101759), (3) the Swedish Governmental Agency for Innovation Systems (VINNOVA) (2013-04655 and 2017-00180), (4) Carl Tryggers Stiftelse för Vetenskaplig Forskning (11:147) and (5) Centre for Antibiotic Resistance Research (CARe) at University

\section{REFERENCES}

Abraham, M. J., Murtola, T., Schulz, R., Páll, S., Smith, J. C., Hess, B., et al. (2015). GROMACS: High performance molecular simulations through multilevel parallelism from laptops to supercomputers. Software X 1-2, 19-25. doi: 10.1016/j.softx.2015.06.001

Allen, S., Zaleski, A., Johnston, J. W., Gibson, B. W., and Apicella, M. A. (2005). Novel sialic acid transporter of Haemophilus influenzae. Infect. Immun. 73, 5291-5300. doi: 10.1128/IAI.73.9.5291-5300.2005

Almagro-Moreno, S., and Boyd, E. F. (2009). Insights into the evolution of sialic acid catabolism among bacteria. BMC Evol. Biol. 9:118. doi: 10.1186/1471-2148-9-118

Baba, T., Ara, T., Hasegawa, M., Takai, Y., Okumura, Y., Baba, M., et al. (2006). Construction of Escherichia coli K-12 in-frame, single-gene knockout mutants: the Keio collection. Mol. Syst. Biol. 2:2006.0008. doi: 10.1038/msb41 00050

Banda, K., Gregg, C. J., Chow, R., Varki, N. M., and Varki, A. (2012). Metabolism of vertebrate amino sugars with $N$-glycolyl groups: mechanisms underlying gastrointestinal incorporation of the non-human sialic acid xenoautoantigen $\mathrm{N}$-glycolylneuraminic acid. J. Biol. Chem. 287, 28852-28864. doi: $10.1074 /$ jbc.M112.364182

Bouchet, V., Hood, D. W., Li, J., Brisson, J.-R., Randle, G. A., Martin, A., et al. (2003). Host-derived sialic acid is incorporated into Haemophilus influenzae lipopolysaccharide and is a major virulence factor in experimental otitis media. PNAS 100, 8898-8903. doi: 10.1073/pnas.1432026100

Brookes, E. H., and Demeler, B. (2007). "Parsimonious regularization using genetic algorithms applied to the analysis of analytical ultracentrifugation experiments," in GECCO '07 Proceedings of the 9th annual Conference on Genetic and Evolutionary Computation (New York, NY).

Byres, E., Paton, A. W., Paton, J. C., Löfling, J. C., Smith, D. F., Wilce, M. C., et al. (2008). Incorporation of a non-human glycan mediates human susceptibility to a bacterial toxin. Nature 456, 648-652. doi: 10.1038/nature07428

Chang, D.-E., Smalley, D. J., Tucker, D. L., Leatham, M. P., Norris, W. E., Stevenson, S. J., et al. (2004). Carbon nutrition of Escherichia coli in the mouse intestine. PNAS 101, 7427-7432. doi: 10.1073/pnas.03078 88101

Demeler, B. (2010). Methods for the design and analysis of sedimentation velocity and sedimentation equilibrium experiments with proteins. Curr. Protoc. Protein. Sci. Chapter 7, Unit 7.13. doi: 10.1002/0471140864.ps0713s60

Demeler, B., and Brookes, E. (2007). Monte Carlo analysis of sedimentation experiments. Coll. Polym. Sci. 286, 129-137. doi: 10.1007/s00396-007-1699-4. of Gothenburg. RN acknowledges EMBO (584-2014). WW acknowledges the Swedish Research Council Formas (221-2013730), Magnus Bergvalls Stiftelse (2014-00536, 2015-00763, 201601606). SR acknowledges the Indo-Swedish grant awarded by the Department of Biotechnology (BT/IN/Sweden/41/SR/2013). JA acknowledges the following for funding support: (1) a Rutherford Discovery Fellowship (15-MAU-001) and (2) the Marsden Fund Council (15-UOA-105). We thank Dr. Mitja Remus-Emsermann (University of Canterbury), for support, guidance, and use of equipment for the bacterial growth assays. We thank Prof. Borries Demeler (University of Texas Health Science Center at San Antonio), for assistance in the analytical ultracentrifugation analysis.

\section{SUPPLEMENTARY MATERIAL}

The Supplementary Material for this article can be found online at: https://www.frontiersin.org/articles/10.3389/fchem. 2018.00233/full\#supplementary-material
Demeler, B., and van Holde, K. E. (2004). Sedimentation velocity analysis of highly heterogeneous systems. Anal. Biochem. 335, 279-288. doi: 10.1016/j.ab.2004.08.039

Hsieh, J. M., Besserer, G. M., Madej, M. G., Bui, H.-Q., Kwon, S., and Abramson, J. (2010). Bridging the gap: a GFP-based strategy for overexpression and purification of membrane proteins with intra and extracellular C-termini. Protein Sci. 19, 868-880. doi: 10.1002/pro.365

Huang, Y.-L., Chassard, C., Hausmann, M., von Itzstein M., and Hennet, T. (2015). Sialic acid catabolism drives intestinal inflammation and microbial dysbiosis in mice. Nat. Commun. 6:8141. doi: 10.1038/ncomms 9141

Jeong, H. G., Oh, M. H., Kim, B. S., Lee, M. Y., Han, H. J., and Choi, S. H. (2009). The capability of catabolic utilization of $N$-acetylneuraminic acid, a sialic acid, is essential for Vibrio vulnificus pathogenesis. Infect. Immun. 77, 3209-3217. doi: 10.1128/IAI.00109-09

Kelm, S., Shi, J., and Deane, C. M. (2010). MEDELLER: homology-based coordinate generation for membrane proteins. Bioinformatics 26, 2833-2840. doi: 10.1093/bioinformatics/btq554

Khan, Z. M., Liu, Y., Neu, U., Gilbert, M., Ehlers, B., Feizi, T., et al. (2014). Crystallographic and glycan microarray analysis of human polyomavirus 9 VP1 identifies $N$-glycolyl neuraminic acid as a receptor candidate. J. Virol. 88, 6100-6111. doi: 10.1128/JVI.03455-13

Larkin, M. A., Blackshields, G., Brown, N. P., Chenna, R., McGettigan, P. A., McWilliam, H., et al. (2007). Clustal W and Clustal X version 2.0. Bioinformatics 23, 2947-2948. doi: 10.1093/bioinformatics/btm404

Laskowski, R. A., and Swindells, M. B. (2011). LigPlot+: multiple ligand-protein interaction diagrams for drug discovery. J. Chem. Inf. Model. 51, 2778-2786. doi: $10.1021 / \mathrm{ci} 200227 \mathrm{u}$

Laue, T. M., Shah, B. D., Ridgeway, T. M., and Pelletier, S. L. (1992). Analytical Ultracentrifugation in Biochemistry and Polymer Science. Cambridge: The Royal Society of Chemistry.

Lee, C., Kang, H. J., Hjelm, A., Qureshi, A. A., Nji, E., Choudhury, H., et al. (2014). MemStar: a one-shot Escherichia coli-based approach for highlevel bacterial membrane protein production. FEBS Lett. 588, 3761-3769. doi: 10.1016/j.febslet.2014.08.025

Myers, E. W., and Miller, W. (1988). Optimal alignments in linear space. Comput. Appl. Biosci. 4, 11-17.

Ng, K. M., Ferreyra, J. A., Higginbottom, S. K., Lynch, J. B., Kashyap, P. C., Gopinath, S., et al. (2013). Microbiota-liberated host sugars facilitate post-antibiotic expansion of enteric pathogens. Nature 502, 96-99. doi: 10.1038 /nature 12503 
North, R. A., Horne, C. R., Davies, J. S., Remus, D. M., Muscroft-Taylor, A. C., Goyal, P., et al. (2017). "Just a spoonful of sugar": import of sialic acid across bacterial cell membranes. Biophys. Rev. 19:425. doi: 10.1007/s12551-017-0343-x

North, R. A., Kessans, S. A., Atkinson, S. C., Suzuki, H., Watson, A. J. A., Burgess, B. R., et al. (2013). Cloning, expression, purification, crystallization and preliminary X-ray diffraction studies of $N$-acetylneuraminate lyase from methicillin-resistant Staphylococcus aureus. Acta Crystallogr. F Struct. Biol. Commun. 69, 306-312. doi: 10.1107/S1744309113003060

North, R. A., Kessans, S. A., Griffin, M. D. W., Watson, A. J. A., Fairbanks, A. J., and Dobson, R. C. J. (2014a). Cloning, expression, purification, crystallization and preliminary X-ray diffraction analysis of $\mathrm{N}$-acetylmannosamine-6-phosphate 2epimerase from methicillin-resistant Staphylococcus aureus. Acta Crystallogr. F Struct. Biol. Commun. 70, 650-655. doi: 10.1107/S2053230X14007171

North, R. A., Seizova, S., Stampfli, A., Kessans, S. A., Suzuki, H., Griffin, M. D. W., et al. (2014b). Cloning, expression, purification, crystallization and preliminary $\mathrm{X}$-ray diffraction analysis of $\mathrm{N}$-acetylmannosamine kinase from methicillinresistant Staphylococcus aureus. Acta Crystallogr. F Struct. Biol. Commun. 70, 643-649. doi: 10.1107/S2053230X14007250

North, R. A., Watson, A. J. A., Pearce, F. G., Muscroft-Taylor, A. C., Friemann, R., Fairbanks, A. J., et al. (2016). Structure and inhibition of $N$ acetylneuraminate lyase from methicillin-resistant Staphylococcus aureus. FEBS Lett. 590, 4414-4428. doi: 10.1002/1873-3468.12462

Olson, M. E., King, J. M., Yahr, T. L., and Horswill, A. R. (2013). Sialic acid catabolism in Staphylococcus aureus. J. Bact. 195, 1779-1788. doi: 10.1128/JB.02294-12

Pezzicoli, A., Ruggiero, P., Amerighi, F., Telford, J. L., and Soriani, M. (2012). Exogenous sialic acid transport contributes to group B streptococcus infection of mucosal surfaces. J. Infect. Dis. 206, 924-931. doi: 10.1093/infdis/jis451

Post, D. M., Mungur, R., Gibson, B. W., and Munson, R. S. (2005). Identification of a novel sialic acid transporter in Haemophilus ducreyi. Infect. Immun. 73, 6727-6735. doi: 10.1128/IAI.73.10.6727-6735.2005

Scalise, M., Pochini, L., Panni, S., Pingitore, P., Hedfalk, K., and Indiveri, C. (2014). Transport mechanism and regulatory properties of the human amino acid transporter ASCT2 (SLC1A5). Amino Acids 46, 2463-2475. doi: 10.1007/s00726-014-1808-x

Scalise, M., Pochini, L., Pingitore, P., Hedfalk, K., and Indiveri, C. (2015). Cysteine is not a substrate but a specific modulator of human ASCT2 (SLC1A5) transporter. FEBS Lett. 589, 3617-3623. doi: 10.1016/j.febslet.2015.10.011

Severi, E., Hosie, A. H., Hawkhead, J. A., and Thomas, G. H. (2010). Characterization of a novel sialic acid transporter of the sodium solute symporter (SSS) family and in vivo comparison with known bacterial sialic acid transporters. FEMS Microbiol. Lett. 304, 47-54. doi: 10.1111/j.1574-6968.2009.01881.x

Severi, E., Randle, G., Kivlin, P., Whitfield, K., Young, R., Moxon, R., et al. (2005). Sialic acid transport in Haemophilus influenzae is essential for lipopolysaccharide sialylation and serum resistance and is dependent on a novel tripartite ATP-independent periplasmic transporter. Mol. Microbiol. 58, 1173-1185. doi: 10.1111/j.1365-2958.2005.04901.x

Soares da Costa, T. P., Desbois, S., Dogovski, C., Gorman, M. A., Ketaren, N. E., Paxman, J. J., et al. (2016). Structural determinants defining the allosteric inhibition of an essential antibiotic target. Structure 24, 1282-1291. doi: 10.1016/j.str.2016.05.019
Stencel-Baerenwald, J. E., Reiss, K., Reiter, D. M., Stehle, T., and Dermody, T. S. (2014). The sweet spot: defining virus-sialic acid interactions. Nat. Rev. Microbiol. 12, 739-749. doi: 10.1038/nrmicro3346

Stifter, S. A., Matthews, A. Y., Mangan, N. E., Fung, K. Y., Drew, A., Tate, M. D., et al. (2018). Defining the distinct, intrinsic properties of the novel type I interferon, IFNє. J. Biol. Chem. 293, 3168-3179. doi: 10.1074/jbc.M117. 800755

Tangvoranuntakul, P., Gagneux, P., Diaz, S., Bardor, M., Varki, N., Varki, A., et al. (2003). Human uptake and incorporation of an immunogenic nonhuman dietary sialic acid. PNAS 100, 12045-12050. doi: 10.1073/pnas.21315 56100

Varki, A. (2001). Loss of $N$-glycolylneuraminic acid in humans: mechanisms, consequences, and implications for hominid evolution. Am. J. Phys. Anthropol. (Suppl. 33), 54-69. doi: 10.1002/ajpa.10018

Varki, A. (2010). Uniquely human evolution of sialic acid genetics and biology. PNAS 107, 8939-8946. doi: 10.1073/pnas.0914634107

Varki, N. M., Strobert, E., Dick, E. J., Benirschke, K., and Varki, A. (2011). Biomedical differences between human and nonhuman hominids: potential roles for uniquely human aspects of sialic acid biology. Annu. Rev. Pathol. 6, 365-393. doi: 10.1146/annurev-pathol-011110-130315

Vimr, E., Lichtensteiger, C., and Steenbergen, S. (2000). Sialic acid metabolism's dual function in Haemophilus influenzae. Mol. Microbiol. 36, 1113-1123. doi: 10.1046/j.1365-2958.2000.01925.x

Vimr, E. R., Kalivoda, K. A., Deszo, E. L., and Steenbergen, S. M. (2004). Diversity of microbial sialic acid metabolism. Microbiol. Mol. Biol. Rev. 68, 132-153. doi: 10.1128/MMBR.68.1.132-153.2004

Vimr, E. R., and Troy, F. A. (1985). Identification of an inducible catabolic system for sialic acids (nan) in Escherichia coli. J. Bact. 164, 845-853.

Wahlgren, W. Y., Dunevall, E., North, R. A., Paz, A., Scalise, M., Bisignano, P., et al. (2018). Substrate-bound outward-open structure of a $\mathrm{Na}^{+}$-coupled sialic acid symporter reveals a novel $\mathrm{Na}^{+}$site. Nat. Commun. 9:1753. doi: 10.1038/s41467-018-04045-7

Wienken, C. J., Baaske, P., Rothbauer, U., Braun, D., and Duhr, S. (2010). Proteinbinding assays in biological liquids using microscale thermophoresis. Nat Commun. 1:100. doi: 10.1038/ncomms1093

Yu, X., Dang, V. T., Fleming, F. E., von Itzstein M., Coulson, B. S., and Blanchard, H. (2012). Structural basis of rotavirus strain preference toward $N$-acetylor N-glycolylneuraminic acid-containing receptors. J. Virol. 86, 13456-13466. doi: 10.1128/JVI.06975-11

Conflict of Interest Statement: The authors declare that the research was conducted in the absence of any commercial or financial relationships that could be construed as a potential conflict of interest.

Copyright (C) 2018 North, Wahlgren, Remus, Scalise, Kessans, Dunevall, Claesson, Soares da Costa, Perugini, Ramaswamy, Allison, Indiveri, Friemann and Dobson. This is an open-access article distributed under the terms of the Creative Commons Attribution License (CC BY). The use, distribution or reproduction in other forums is permitted, provided the original author(s) and the copyright owner(s) are credited and that the original publication in this journal is cited, in accordance with accepted academic practice. No use, distribution or reproduction is permitted which does not comply with these terms. 